\title{
From classical Langerhans cell histiocytosis to Erdheim- -Chester disease: different sides of the same coin?
}

\author{
Elżbieta Grześk (D), Anna Dąbrowska, Sylwia Kołtan, Mariusz Wysocki, Krzysztof Czyżewski, \\ Jan Styczyński (D)
}

Department of Pediatric Hematology and Oncology, Collegium Medicum in Bydgoszcz, Nicolaus Copernicus University in Toruń, Jurasz University Hospital 1, Bydgoszcz, Poland

\begin{abstract}
Introduction: We analysed the clinical course of classical histiocytosis (LCH) in children, and the clinical differences, diagnostic difficulties and different therapeutic strategy in a child with a rare variant of LCH in the form of ErdheimChester disease (ECD).
\end{abstract}

Material and methods: We conducted a retrospective single-center analysis of the clinical course of classic LCH in 54 children who were diagnosed with the disease over the last 40 years, and the differences shown in a patient with diagnosed ECD. The clinical response was assessed according to the LCH programs valid at the time for the classic form of $\mathrm{LCH}$ and in the child with ECD.

Results: The multi-system form of LCH was diagnosed more often than the single-system form. The skull bones were the most common localization of LCH in both forms of the disease. Recurrence of the disease occurred in about $5 \%$ of patients, and death in one (1.9\%) patient. The course of the child with ECD was more turbulent, with rapid progression, the involvement of critical organs, and no response to standard chemotherapy according to the LCH 2009 protocol. After a molecular diagnosis was specified, therapy with vemurafenib, a BRAF-V600E kinase inhibitor, followed by allogeneic hematopoietic stem cells transplantation (allo-HSCT) was applied. The basic disease has been in remission for the last 12 months.

Conclusions: The lack of an expected response to LCH therapy should indicate the possibility of rare forms of histiocytic hyperplasia. Molecular tests are an important element in the diagnosis of histiocytosis, and allow the precise selection of the most appropriate, targeted therapy. BRAF-V600E kinase inhibitors are highly safe and effective in the treatment of LCH and ECD with a confirmed BRAF-V600E mutation, although allo-HSCT should be considered in selected cases.

Key words: Erdheim-Chester disease, BRAF-V600E mutation, vemurafenib, children

Acta Haematologica Polonica 2021; 52, 6: 571-577

\section{Introduction}

Langerhans cell histiocytosis ( $\mathrm{LCH}$ ) is a very rare disease, with a frequency of 0.1-1 new case per 100,000 children per year. Most often it is diagnosed up to the age of 6 [1]. $\mathrm{LCH}$ is a heterogeneous group of diseases characterized by uncontrolled growth, proliferation and differentiation of cells of the mononuclear-phagocytic system. It belongs to the family of clonal proliferative diseases, and lies on the border with neoplastic diseases. At the root of LCH are disturbances in the mitogen-activated kinase (MAPK) pathway. Their occurrence is associated with a more serious course

\footnotetext{
*Address for correspondence: Elżbieta Grześk, Department of Pediatric Hematology and Oncology, Collegium Medicum, Nicolaus Copernicus University Toruń, Skłodowskiej-Curie 9, 85-094 Bydgoszcz, Poland, e-mail: ellag@cm.umk.pl

\footnotetext{
PTHiT Copyright (c) 2021

The Polish Society of Haematologists and Transfusiologists, Insitute of Haematology and Transfusion Medicine. All rights reserved.
} 
and a higher probability of progression and recurrence. One of the rare forms of histiocytic hyperplasia in children is Erdheim-Chester disease (ECD). ECD's pathogenesis is related to the BRAF-V600 mutation [1, 2].

Histiocytosis can affect any organ or system, with the occurrence of permanent complications in $20-30 \%$ of patients, including death. The skeletal system (80\%), skin (33\%) and then the pituitary gland (25\%) are most often involved. The disease is also localized in parenchymal organs: liver, spleen, hematopoietic system and lungs (15\% each), lymph nodes (5-10\%) and the central nervous system excluding the pituitary gland (2-4\%). The clinical course varies from self-limited to rapidly progressive, leading to death.

Treatment varies according to the severity of the disease. The response to first-line treatment is important prognostic information, and helps in the selection of a further therapeutic strategy, often based on genetic tests, allowing for the selection of the most effective therapy [3-6]. The number of randomized clinical trials is limited in the literature, and many aspects of management of this condition remain controversial.

The current diagnostic procedure in pediatric $\mathrm{LCH}$ has been developed by the Histiocytic Society and also based on evidence-based-medicine papers, published between 2009 and 2020 [4, 7, 8]. In the diagnosis of histiocytosis, a histopathological examination with immunophenotyping is decisive and differentiating. The current guidelines suggest mandatory genetic testing to detect mutations in the MAPK pathway, especially the mutation identifying BRAF. Clinical and laboratory data allows the patient to be classified as either single system or multi system, as well as to determine the involvement of critical organs. Basic tests include blood tests (hematological, biochemical, liver and kidney function, coagulation parameters and lipid profile), urine tests, and imaging tests (lung X-ray, abdominal ultrasound). Scintigraphy of the skeletal system, as well as positron emission tomography with computed tomography (PET-CT) in patients with indications for systemic treatment, is very important. Such meticulous diagnostics allows all affected organs to be identified and facilitates monitoring of the response to treatment. The study designs in $\mathrm{LCH}$ patients have been presented many times and are consistent in different countries, according to different working groups [4-6].

$\mathrm{LCH}$ in the past, the mysterious histiocytosis $\mathrm{X}$, still eludes exhaustive classification. The most recent classification divides disorders in the histiocytosis group into five groups (Tables I and II): LCH, ECD, indeterminate cell histiocytosis (ICH), extra-cutaneous JXG, as well as mixed forms of $L C H$ and ECD belonging to the group $L$ histiocytosis $[3,4,9,10]$.

Erdheim-Chester disease is a rare non-Langerhans cell histiocytosis (non-LCH), first described as a "lipid granulomatosis" by Jakob Erdheim and William Chester in
Table I. Classification of histiocytoses (source [9])

\begin{tabular}{|c|c|}
\hline $\begin{array}{l}\text { Histiocytosis } \\
\text { group }\end{array}$ & Diseases \\
\hline $\mathrm{L}$ & $\begin{array}{l}\text { LCH } \\
\text { Indeterminate-cell histiocytosis (ICH) } \\
\text { Erdheim-Chester disease (ECD) } \\
\text { Mixed LCH/ECD }\end{array}$ \\
\hline C & $\begin{array}{l}\text { Cutaneous non-LCH } \\
\text { Xanthomatous granuloma (XG) family: JXG, } \\
\text { AXG, SRH, BCH, GEH, PNH } \\
\text { Non-XG family: cutaneous RDD, NXG, other } \\
\text { Cutaneous non-LCH with a major systemic } \\
\text { component } \\
\text { XG family: XD } \\
\text { Non-XG family: MRH }\end{array}$ \\
\hline $\mathrm{R}$ & $\begin{array}{l}\text { Familial RDD } \\
\text { Sporadic RDD } \\
\text { Classical RDD } \\
\text { Extranodal RDD } \\
\text { RDD with neoplasia or immune disease } \\
\text { Unclassified }\end{array}$ \\
\hline$M$ & $\begin{array}{l}\text { Primary malignant histiocytoses } \\
\text { Secondary malignant histiocytoses }\end{array}$ \\
\hline $\mathrm{H}$ & $\begin{array}{l}\text { Primary HLH: monogenic inherited conditions } \\
\text { leading to HLH } \\
\text { Secondary HLH (non-Mendelian HLH) } \\
\text { HLH of unknown or uncertain origin }\end{array}$ \\
\hline
\end{tabular}

1930 [11]. So far, about 1,500 cases of ECD have been reported worldwide, but only 11 in children [12]. The age at onset of the disease is $43-55$ years, and it is more common in men [13-16]. Average survival time in adults is c. 32 months [17]. ECD is a multi-system clonal hematopoietic disease which comprises the infiltration of tissues by histiocytes taking on the characteristic appearance of foam cells [18-20]. Somatic genetic mutations are responsible for clonal growth of the bone marrow, as a result of which the MAPK pathways are activated in the inflammatory environment $[7,19]$. The most common mutation in the MAPK pathway in ECD is the BRAF-V600E mutation (60-100\% of patients) [13, 16, 20-22]. Other mutations are PIK3CA in $11 \%$ and NRAS in $4 \%$ of patients [23]. A strong systemic inflammatory reaction is associated with the activation of the Th1-dependent response, resulting in the production 
Table II. Proposed new classification of histiocytosis group L (acc. to [10])

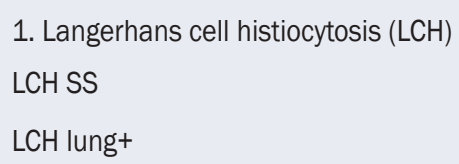

of the cytokines interferon (IFN) alpha, interleukin (IL) 1/ /IL-1 receptor antagonist IL-1-RA, IL-6, IL-12 and monocyte chemoattractant protein 1 (MCP-1) [7, 19].

In a typical histopathological presentation, in addition to foam histiocytes, giant Tauton cells and stromal fibrosis are common. In immunohistochemistry, histiocytes show positive reactions for CD68, CD163 and factor XIIla, negative for CD1a and langerin. Most commonly, the S100 protein is not detected. Unlike ECD, histiocytic cells in LCH have the phenotype of CD1a+, S100+ and Langerin+ [15, 19, 20]. Histopathological and immunohistochemical images in ECD and in juvenile xanthogranuloma (JXG) are almost the same, and therefore it is believed that ECD is a variant of JXG but without dominant skin involvement [20]. In the course of ECD, any organ may be involved, most often the long bones (in $80-90 \%$ of patients), the retroperitoneal space within the kidneys (60\%), the cardiovascular system $(50 \%+)$, the central nervous system $(40-50 \%)$, the lungs (15-33\%), and less commonly the skin, lymph nodes, liver, and spleen [1, 5, 12, 23-28]. The multi-organ form of ECD consists of skeletal failure, with the most common symmetrical bilateral osteosclerotic changes in the long bones around the knee joints.

Bone pain occurs in 39\% of patients, and is localized in the distal sections of the lower limbs [28]. In children, the involvement of the skull, mandible, ribs and spine bones is more common [2]. In the multi-system form, the next affected organ is the kidneys, giving the typical image of infiltrates in computed tomography, the so-called 'hairy kidney'. Cardiovascular symptoms in ECD may include a pseudo-tumor in the right atrium, changes in the coronary arteries, and pericarditis. Lung involvement is frequent, with cellular infiltration and thickening of the alveoli with pleural effusion. Swelling of the optic nerve and the presence of retrobulbar masses can cause exophthalmos and impaired motor function, as well as fibrotic changes along the optic nerve, extending up to the hypothalamus and pituitary gland, with the possibility of diabetes insipidus. Yellow tufts (xanthelasma), as well as yellow or red-brown foci, may appear on the skin $[11,16,20,25,27,29]$.

The first ECD therapies were based on chemotherapy (vinblastine, cyclophosphamide, anthracyclines), radiotherapy, steroid therapy, interferon alpha, and anti-cytokine drugs (anakinra, infliximab and tocilizumab), and showed improvement in only $50 \%$ of patients $[11,12,16]$. The discovery and frequent occurrence of the BRAF-V600E mutation in ECD revolutionized the understanding of the pathogenesis of the disease and led to research focused on appropriate therapies showing high efficacy [15, 30]. In 2012, the BRAF and extracellular-signal regulated kinase (MEK) inhibitors, vemurafenib and cobimetinib, respectively, were introduced to the treatment of ECD, and in 2017 the US Food and Drug Administration (FDA) approved vemurafenib for the treatment of BRAF+ ECD [11]. Anti-BRAF therapy (vemurafenib) is currently recommended at diagnosis in all BRAF+ patients, except for asymptomatic patients $[7,19,20]$ (Figure 1). BRAF and MEK inhibitors are believed to be effective and safe drugs in life-threatening forms of ECD $[3,4,5,9]$. The prognosis for ECD is generally good, but there are cases where second-line treatment and hematopoietic cell transplantation are required.

Due to the rarity of $\mathrm{LCH}$, each initiation of therapy requires verification not only of the current literature but also of the available pharmacotherapy, in both registration and off-label modes. In the absence of clearly defined management protocols, it is also necessary to perform a retrospective evaluation of the effectiveness and safety of the applied therapy in previously treated patients.

The aim of our study was to analyze the clinical course and treatment effects of classic $\mathrm{LCH}$, diagnosed in children over the last 40 years at our Clinic, and to analyze the course and treatment of ECD in a 3-year-old girl with a critical analysis of the similarities and differences in both forms of histiocytic hyperplasia.

\section{Material and methods}

Our study consisted of two parts. Firstly, we looked at the epidemiology and treatment outcomes of LCH in children in a 40-year (1980-2020) single center study. Medical records concerning hospitalization and outpatient treatment of patients were assessed. Due to the limited access to historical documentation, it was possible to obtain only some clinical data. Secondly, we assessed the diagnostic process in a patient with atypical symptoms and initial lack of response to treatment according to the classic LCH 


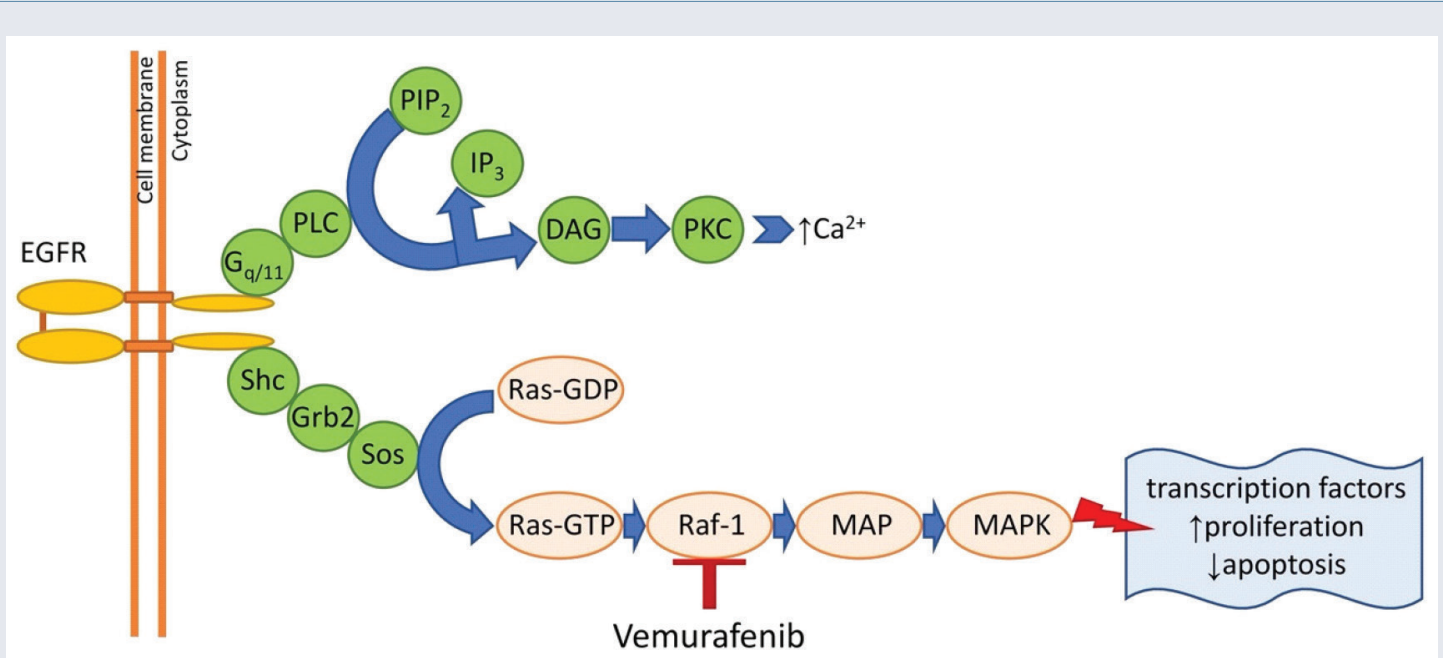

Figure 1. Schematic illustration of vemurafenib intervention in proliferation and apoptosis process; $\mathrm{Ca}^{2+}$ - calcium ions; $\mathrm{DAG}$ - diacylglycerol; EGFR - endothelial growth factor receptor; GDP - guanosine diphosphate; GTP - guanosine triphosphate; GRB2 - growth factor receptorbound protein 2; $\mathrm{G}_{\mathrm{q} / 11}-\mathrm{G}$ protein type $\mathrm{G}_{\mathrm{q} / 11} ; \mathrm{IP}_{3}$ - inositol 1,4,5-trisphosphate; MAP - mitogen-activated protein; MAPK - mitogen-activated protein kinase; $\mathrm{PIP}_{2}$ - phosphatidylinositol 4,5-bisphosphate; PLC - phospholipase type C; PKC - protein kinase type C; RAF-1 - serine/ /threonine-specific protein kinases; RAS - small GTPase; Shc - signaling adaptor protein; Sos - guanosine nucleotide exchange factor

treatment protocol, the importance of molecular tests for correct diagnosis, and the effects of targeted treatment with the subsequent option of hematopoietic cell transplantation.

\section{Results}

\section{Epidemiology, clinical manifestation and results of LCH treatment in children 1980-2020}

Between 1980 and 2020, 1,785 children were diagnosed and treated at the Department of Pediatrics, Hematology and Oncology, Nicolaus Copernicus University in Toruń, Bydgoszcz, Poland. The number of patients with hematological malignancies (acute lymphoblastic leukemia (ALL), acute myeloblastic leukemia (AML), myelodysplastic syndrome (MDS), chronic myeloid leukemia (CML), non-Hodgkin lymphoma (NHL), Hodgkin lymphoma $(\mathrm{HL})$, and $\mathrm{LCH}$ was 1,069 (59.9\%), including 54 with $\mathrm{LCH}$, which accounts for $3.0 \%$ of all malignant neoplasms and $5.1 \%$ of all hematological tumors.

In the studied group of children, the multi-system form was observed more often (in 54\%, see Table III). The dominant clinical manifestations of LCH in any form were bone lesions located in the bones of the skull in several patients with intracranial penetration and infiltration of the dura mater. Bone lesions also occurred in the eye socket, in the mastoid process, in the ribs, in the mandible, in the long bones, and in one patient in the vertebrae of the thoracic spine and the pubic bone. The next most frequently affected organ was the skin. In the multi-system form, changes in the skeletal system, lungs, liver, spleen, peripheral lymph nodes and pancreas were simultaneously observed. Recurrence of the disease occurred in a total of three patients (5.5\%). One patient died (1.85\%).

The treatment was applied in accordance with the recommendations in force at the time. In recent years, the treatment was implemented according to the Langerhans Cell Histiocytosis 2009 protocol, Histiocyte Society Evaluation and Treatment Guidelines MS-LCH.

In 1/54 patients with primary diagnosed multiorgan $\mathrm{LCH}$, the course was atypical, with no involvement of the skeletal system, but with involvement of the critical organs: liver, spleen and bone marrow. No improvement was achieved after the treatment according to LCH 2009. Due to the disease progression, the diagnostics was extended and a molecular test was performed. The obtained results made it possible to verify the diagnosis at ECD and to implement targeted and effective therapy.

\section{Erdheim-Chester disease: analysis of diagnostic and therapeutic process}

A 3-years-old girl with an unburdened perinatal history, and with a family history of sarcoidosis in the father and inhaled allergy in the mother, was admitted to our department. By the age of 2.5 years, she had been diagnosed with recurrent abdominal pain and diarrhea. Primary malabsorption syndrome and inflammatory disease of the gastrointestinal tract were excluded, and allergy to cow's milk protein and rice confirmed. After introducing the diet, the child's condition improved moderately. At the age of 2.7 years, the child developed a high fever 
Table III. Characteristics of patients with Langerhans cell histiocytosis $(\mathrm{LCH})$

\begin{tabular}{l|l}
\hline Variable & Value \\
\hline Number of patients & $54(100 \%)$ \\
Age (years): median (range) & $6.25(0.5-17.8)$ \\
\hline Sex: female vs. male & $26(48.2 \%) / 28(51.8 \%)$ \\
$\begin{array}{l}\text { Organ involvement: } \\
\text { - bone }\end{array}$ & $44(81.5 \%)$ \\
- skin & $16(30 \%)$ \\
- hematopoietic & $2(3.7 \%)$ \\
- spleen & $2(3.7 \%)$ \\
- liver & $2(3.7 \%)$ \\
- lungs & $2(3.7 \%)$ \\
- eye & $1(1.85 \%)$ \\
- ear & $3(5.5 \%)$ \\
- diabetes insipidus & $5(9.3 \%)$ \\
- central nervous system & $5(9.3 \%)$ \\
- lymph nodes & $3(5.5 \%)$ \\
\hline $\begin{array}{l}\text { Form of disease: } \\
\text { - single-system LCH }\end{array}$ & $25(46 \%)$ \\
- multisystem LCH with or without & $29(54 \%)$ \\
\hline involvement of risk organs &
\end{tabular}

with generalized lymphadenopathy, hepatosplenomegaly and bilinear cytopenia. Bone marrow biopsy revealed hypoplasia and suspected aplastic anemia. Skin purpura, hepatosplenomegaly, and a large, bloated abdomen were observed. The clinical presentation was dominated by severe abdominal pain and diarrhea. Laboratory tests revealed a decreased activity of amylases in blood serum, and in the CT examination, a manifestation of the pancreas typical for organ inflammation. Acute pancreatitis was treated, but there was no improvement. As part of the differential diagnosis, bacterial, viral, fungal and parasitic infections, Gaucher disease, Niemann-Pick disease, sarcoidosis, autoimmune lymphoproliferative syndrome, and phagocytosis disorders were excluded. Due to persistent bone marrow insufficiency and exocrine pancreatic insufficiency, Shwachman-Diamond syndrome was suspected, which was excluded by molecular examination. Myelodysplastic and hemophagocytic syndromes were also excluded. The presence of histiocytes with a foam-like cytoplasm, CD68+, part XIII+, CD1a-, Langerin -, in a few $\mathrm{S}-100+$ cells was found. The bedding showed the loss of reticulin fibers. The image corresponded to a diagnosis of disseminated juvenile xanthogranuloma.

High-resolution computed tomography of the chest (HRCT) revealed inflammatory changes and areas of 'frosted glass', as well as nodules in the lungs that may correspond to infiltrates in the course of histiocytosis. No pathological changes were found in the scintigraphy of the skeletal system. The PET-CT examination showed no signs of an active malignant proliferative process. There were also no deviations in cardiological and neurological examination.
Treatment according to the LCH-2009 protocol (prednisone, vinblastine) was started, after which the progression of clinical symptoms and increasing signs of bone marrow failure were observed. Another bone marrow biopsy test revealed the presence of CD68+, CD1a-, S-100-, CD14+, CD163+ protein, moreover the BRAF-V600E mutation was found in bone marrow cells.

Eventually, ECD was diagnosed. After obtaining the approval of the Therapeutic Committee for off-label therapy, anti-BRAF therapy with the drug vemurafenib at a dose of $10 \mathrm{mg} / \mathrm{kg}$ bw/day was initiated, achieving a spectacular clinical improvement. The symptoms of the gastrointestinal tract subsided, and normalization of blood cell count and biochemical parameters was observed. The parenchymal organs of the abdominal cavity decreased significantly. Normal CT image of the pancreas was observed. Anti-BRAF therapy was used for three months without any side effects. Subsequently, an allogeneic hematopoietic stem cell (allo-HSCT) transplantation was performed from a matched unrelated donor. The post-transplant course was uneventful. In the control tests performed two months after allo-HSCT, no BRAF-V600E mutation was found. The bone marrow image was rich in cells with reconstruction of all lines of the hematopoietic system. Currently, the child is in the 11th month of follow-up after allo-HSCT, in clinical and molecular ECD remission.

\section{Discussion}

Histiocytic hyperplasia is a rare form of cancer in children. In our Clinic, they were diagnosed in 3\% of pediatric patients diagnosed with any neoplastic disease, and in c.5\% of pediatric patients diagnosed with hematological neoplasms. The classic form of LCH is a disease with a good prognosis: 5 -year survival in children is $90 \%$. However, relapses are observed in as many as one third of patients [9]. In our presented study, recurrence of $\mathrm{LCH}$ was reported in 5.5\% of children.

The course of the rare forms of histiocytic hyperplasia, including ECD, is different.

The age at onset was between 1 year and 14 years $[12,14]$. In all children, the skeletal system was affected (long bones, bones of the cranial vault, jaw, mandible, pelvic bones, ribs and spine). Infiltrations in the lungs and retroperitoneal space were noted in two patients, and in the central nervous system in three. One patient had hepatosplenomegaly $[2,14]$. The most common involvement, of the bones, skin, cardiovascular system and central nervous system, was not confirmed in the presented child. Her clinical picture was dominated by bone marrow failure, severe abdominal pain and diarrhea with hypoalbuminemia associated with infiltrates in the pancreas and its exocrine insufficiency. The consequence of bone marrow aplasia was the necessity to use numerous blood transfusions and 
the treatment of life-threatening infections. The presented patient had the clinical manifestation with predominant myelo-pancreatic-pulmonary disease of Erdheim-Chester.

Among the children with ECD described so far, none of them had bone marrow failure $[2,14]$, which was the dominant symptom in our patient. The literature lacks data on the frequency of malignant bone marrow transformation in children with ECD. However, it is known from the literature that ECD is associated with an increased risk of hyperplasia of the hematopoietic system, mainly the myeloid lineage [25]. Papo et al. [26] reported cases of chronic myelomonocytic leukemia, myelodysplastic and myeloproliferative syndromes, and polycythemia vera in $10 \%$ of adult ECD patients. In a study by Cohen-Aubart et al. [19], $15.8 \%$ of adult ECD patients developed myeloid neoplasm with present genetic abnormalities in the TET2, ASXL1, DNMT3A, and NRAS genes. These patients were significantly more often elderly, and the BRAF-V600E mutation was found significantly more often [19].

In the case reports of childhood ECD, delays in diagnosis of the disease, ranging from six months to six years, are frequently underscored [2]. It cannot be ruled out that the symptoms of the disease had appeared in our patient much earlier, as the girl had had abdominal pain and recurrent diarrhea from age 9 months. The causes of the ailments were not established in the extensive diagnostics, and dietary treatment did not bring about any significant improvement. For a year before the correct diagnosis, the girl was treated with pancreatin, with quite good results. This would suggest some initial changes in the pancreas. In the diagnosis of histiocytosis, a biopsy of the affected organ should be carried out together with the assessment of the MAPK pathway mutation. Due to the similarities between ECD, JXG and LCH, it is sometimes necessary to repeat the histopathological examination, especially when the patient does not respond to the treatment according to the $\mathrm{LCH}$ protocol [14]. This was the case in the described patient, who initially received treatment with vinblastine and steroids, but, due to disease progression, another bone marrow trephine biopsy was performed with the assessment of the BRAF-V600E mutation.

Confirmation of the BRAF-V600E mutation firstly differentiates ECD from JXG, and secondly allows for an effective targeted therapy. Among 11 reported children with ECD, only two were tested for the BRAF-V600E mutation, which was confirmed in one. MAPK inhibitors were not used in the therapy [14]. The literature also lacks data on hematopoietic cell transplantation in patients with ECD. The presented patient was the first child to develop bone marrow failure in the course of ECD. Taking into account the lack of data on anti-BRAF therapy in children worldwide (duration of therapy, side effects, effectiveness) and the documented risk of bone marrow malignant transformation, it was decided to transplant the patient with allogeneic hematopoietic cells with preceding anti-BRAF therapy. This treatment led to a complete recovery.

\section{Conclusions}

Erdheim-Chester disease is extremely rare in children, but it should be considered in all cases of histiocytosis, especially in multi-system forms, with poor response to standard therapy. Molecular diagnostics should be sought. The described case of a child is also an example of the diagnostic difficulties in bone marrow aplasia. The clinical presentation of the patient and the cytomorphological manifestation of the bone marrow initially corresponded to aplastic anemia, but the decisive factor was the histopathological and immunohistochemical results of bone marrow trephine biopsy and confirmation of BRAF-V600E mutation in histiocytes. The heterogeneity and diversity of the clinical symptoms, and the atypical age of the patient were the reasons for the long drawn-out differential diagnosis, but with therapeutic success through the use of anti-BRAF therapy followed by the procedure of hematopoietic cells transplantation.

\section{Authors' contributions}

EG - study design, data collection and analysis, interpretation of results, writing manuscript; JS - study design, critical revision of manuscript; all other Authors contributed to provision of important clinical data, interpretation of results, and final approval.

\section{Conflict of interest}

None.

\section{Financial support}

None.

\section{Ethics}

The work described in this article has been carried out in accordance with The Code of Ethics of the World Medical Association (Declaration of Helsinki) for experiments involving humans; EU Directive 2010/63/EU for animal experiments; Uniform requirements for manuscripts submitted to biomedical journals.

\section{References}

1. Raciborska A, Małas Z, Tysarowski A. [Vemurafenib in refractory langerhans histiocytosis] [Article in Polish]. Dev Period Med. 2018; 22(4): 376-378, indexed in Pubmed: 30636236.

2. Drabko K, Raciborska A. Standard diagnostyki histiocytozy z komórek Langerhansa (Langerhans cell histiocytosis - LCH) w Polsce. Przeg| Pediatr. 2019; 48(3): 72-74.

3. Goyal G, Heaney ML, Collin M, et al. Erdheim-Chester disease: consensus recommendations for evaluation, diagnosis, and treatment in the molecular era. Blood. 2020; 135(22): 1929-1945, doi: 10.1182/ blood.2019003507, indexed in Pubmed: 32187362. 
4. Haroche J, Cohen-Aubart F, Amoura Z. Erdheim-Chester disease. Blood. 2020; 135(16): 1311-1318, doi: 10.1182/blood.2019002766.

5. Arnaud L, Hervier B, Néel A, et al. CNS involvement and treatment with interferon- $\alpha$ are independent prognostic factors in Erdheim-Chester disease: a multicenter survival analysis of 53 patients. Blood. 2011; 117(10): 2778-2782, doi: 10.1182/blood-2010-06-294108, indexed in Pubmed: 21239701.

6. Hervier B, Haroche J, Arnaud L, et al. French Histiocytoses Study Group. Association of both Langerhans cell histiocytosis and Erdheim-Chester disease linked to the BRAFV600E mutation. Blood. 2014; 124(7): 1119-1126, doi: 10.1182/blood-2013-12-543793, indexed in Pubmed: 24894769.

7. Nagatsuka H, Han PP, Taguchi K, et al. Erdheim-Chester disease in a child presenting with multiple jaw lesions. J Oral Pathol Med. 2005; 34(7): 420-422, doi: 10.1111/j.1600-0714.2005.00320.x, indexed in Pubmed: 16011611.

8. Haroche J, Cohen-Aubart F, Rollins BJ, et al. Histiocytoses: emerging neoplasia behind inflammation. Lancet Oncol. 2017; 18(2): e113-e125, doi: 10.1016/S1470-2045(17)30031-1, indexed in Pubmed: 28214412.

9. Rodriguez-Galindo C, Allen C. Langerhans cell histiocytosis. Blood. 2020; 135(16): 1319-1331, doi: 10.1182/blood.2019000934.

10. Radzikowska E. Pulmonary Langerhans' cell histiocytosis in adults. Adv Respir Med. 2017; 85(5): 277-289, doi: 10.5603/ARM. a2017.0046, indexed in Pubmed: 29083024.

11. Diamond EL, Dagna L, Hyman DM, et al. Consensus guidelines for the diagnosis and clinical management of Erdheim-Chester disease. Blood. 2014; 124(4): 483-492, doi: 10.1182/blood-2014-03-561381, indexed in Pubmed: 24850756.

12. Khan MR, Ashraf M, Belgaumi A. Erdheim Chester disease - an unusual presentation of a rare histiocytic disease in a 3-year old boy. Pediatric Hematology Oncology Journal. 2017; 2(3): 59-62, doi: 10.1016/j.phoj.2017.09.004.

13. Haroche J, Arnaud L, Cohen-Aubart F, et al. Erdheim-Chester disease. Curr Rheumatol Rep. 2014; 16(4): 412, doi: 10.1007/s11926-0140412-0, indexed in Pubmed: 24532298.

14. McClain K. Histiocytic disorders: insights into novel biology and implications for therapy of Langerhans cell histiocytosis and Erdheim-Chester disease. Hematology Am Soc Hematol Educ Program. 2020; 2020(1): 395-399, doi: 10.1182/hematology.2020000159, indexed in Pubmed: 33275721.

15. Starkebaum G, Hendrie P. Erdheim-Chester disease. Best Pract Res Clin Rheumatol. 2020; 34(4): 101510, doi: 10.1016/j. berh.2020.101510, indexed in Pubmed: 32305313.

16. Haroche J, Abla O. Uncommon histiocytic disorders: Rosai-Dorfman, juvenile xanthogranuloma, and Erdheim-Chester disease. Hematology Am Soc Hematol Educ Program. 2015; 2015: 571-578, doi: 10.1182/ asheducation-2015.1.571, indexed in Pubmed: 26637774.

17. Picarsic J, Pysher T, Zhou H, et al. BRAF V600E mutation in juvenile xanthogranuloma family neoplasms of the central nervous system (CNS-JXG): a revised diagnostic algorithm to include pediatric Erdheim-Chester disease. Acta Neuropathol Commun. 2019; 7(1): 168, doi: 10.1186/s40478-019-0811-6, indexed in Pubmed: 31685033.

18. Cohen Aubart F, Roos-Weil D, Armand M, et al. High frequency of clonal hematopoiesis in Erdheim-Chester disease. Blood. 2021; 137(4):
485-492, doi: 10.1182/blood.2020005101, indexed in Pubmed: 33067622.

19. Cohen-Aubart F, Emile JF, Carrat F, et al. Phenotypes and survival in Erdheim-Chester disease: results from a 165-patient cohort. Am J Hematol. 2018; 93(5): E114-E117, doi: 10.1002/ajh.25055, indexed in Pubmed: 29396850.

20. Lachenal F, Cotton F, Desmurs-Clavel H, et al. Neurological manifestations and neuroradiological presentation of Erdheim-Chester disease: report of 6 cases and systematic review of the literature. J Neurol. 2006; 253(10): 1267-1277, doi: 10.1007/s00415-006-0160-9, indexed in Pubmed: 17063320.

21. Arnaud L, Pierre I, Beigelman-Aubry C, et al. Pulmonary involvement in Erdheim-Chester disease: a single-center study of thirty-four patients and a review of the literature. Arthritis Rheum. 2010; 62(11): 3504-3512, doi: 10.1002/art.27672, indexed in Pubmed: 20662053.

22. Goyal G, Young JR, Koster MJ, et al. Mayo Clinic Histiocytosis Working Group. The Mayo Clinic Histiocytosis Working Group Consensus Statement for the Diagnosis and Evaluation of Adult Patients With Histiocytic Neoplasms: Erdheim-Chester Disease, Langerhans Cell Histiocytosis, and Rosai-Dorfman Disease. Mayo Clin Proc. 2019; 94(10): 2054-2071, doi: 10.1016/j.mayocp.2019.02.023, indexed in Pubmed: 31472931.

23. Song SY, Lee SW, Ryu KH, et al. Erdheim-Chester disease with multisystem involvement in a 4-year-old. Pediatr Radiol. 2012; 42(5): 632-635, doi: 10.1007/s00247-011-2235-8, indexed in Pubmed: 21879308.

24. Tran TA, Fabre M, Pariente D, et al. Erdheim-Chester disease in childhood: a challenging diagnosis and treatment. J Pediatr Hematol Oncol. 2009; 31(10): 782-786, doi: 10.1097/MPH.0b013e3181b76827, indexed in Pubmed: 19755920.

25. Cives M, Simone V, Rizzo FM, et al. Erdheim-Chester disease: a systematic review. Crit Rev Oncol Hematol. 2015; 95(1): 1-11, doi: 10.1016/j.critrevonc.2015.02.004, indexed in Pubmed: 25744785.

26. Papo M, Emile JF, Maciel TT, et al. Erdheim-Chester disease: a concise review. Curr Rheumatol Rep. 2019; 21(12): 66, doi: 10.1007/s11926019-0865-2, indexed in Pubmed: 31807955.

27. Campochiaro C, Cavalli G, Farina N, et al. Efficacy and improved tolerability of combination therapy with interleukin-1 blockade and MAPK pathway inhibitors for the treatment of Erdheim-Chester disease. Ann Rheum Dis. 2019 [Epub ahead of print], doi: 10.1136/annrheumdis-2019-216610, indexed in Pubmed: 31818808.

28. Papo M, Diamond EL, Cohen-Aubart F, et al. High prevalence of myeloid neoplasms in adults with non-Langerhans cell histiocytosis. Blood. 2017; 130(8): 1007-1013, doi: 10.1182/blood-2017-01-761718, indexed in Pubmed: 28679734.

29. Haupt R, Minkov M, Astigarraga I, et al. Euro Histio Network. Langerhans cell histiocytosis ( $\mathrm{LCH}$ ): guidelines for diagnosis, clinical workup, and treatment for patients till the age of 18 years. Pediatr Blood Cancer. 2013; 60(2): 175-184, doi: 10.1002/pbc.24367, indexed in Pubmed: 23109216.

30. Váradi Z, Bánusz R, Csomor J, et al. Effective BRAF inhibitor vemurafenib therapy in a 2-year-old patient with sequentially diagnosed Langerhans cell histiocytosis and Erdheim-Chester disease. Onco Targets Ther. 2017; 10: 521-526, doi: 10.2147/0TT.S121615, indexed in Pubmed: 28182116. 